\title{
Perfil dermatoglífico y somatotipificación de jugadores adolescentes defútbol Dermatoglyphic profile and somatotyping of adolescent soccer players
}

*Aldo Neyl Rodriguez, **Oscar Montenegro, *Jorge Luis Petro

*Universidad de Córdoba (Colombia), ${ }^{* *}$ Universidad Surcolombiana (Colombia)

\begin{abstract}
Resumen. El propósito del presente estudio fue determinar el perfil dermatoglífico y la somatotipificación de un grupo de adolescentes (edad, 15.2 \pm 0.3 años) de la selección de futbol del departamento de Córdoba, Colombia. Se realizaron mediciones antropométricas con las cuales se derivó indicadores como el índice de masa corporal (IMC), grasa corporal (GC) y el somatotipo; igualmente, se realizó registro de la huella dactilar y se determinó, siguiendo el protocolo de Cummins y Midlo (1942), los diseños digitales: Arco (A), Presilla (L) y Verticilo (W), Índice Delta (D10), sumatoria de la cantidad total de líneas (SQTL) y, adicionalmente, los tipos de fórmulas digitales (AL, ALW, 10L, 10W, LW y WL). Los resultados de los indicadores antropométricos fueron: IMC $=20.5 \pm 2.1 \mathrm{~kg}^{\prime} » \mathrm{~m}^{2}$, GC $=10.2 \pm 2.7 \%$; los valores encontrados en los componentes del somatotipo fueron de $3.4 \pm 0.5$ - $4.6 \pm 0.9-3.4 \pm 1.1$ (endomorfía - mesomorfía - ectomorfía, respectivamente). Con relación a los indicadores dermatoglíficos, se encontró una presencia de: $\mathrm{A}=5 \%, \mathrm{~L}=65 \%$ y W $=31 \%$; el D10 = $12.6 \pm 3.5$ y la SQTL $=131.7 \pm 39.1$. Por otra parte, el predominio de fórmulas digitales fue de LW (40\%), seguido de WL (20\%) y ALW y 10L (con el 15\% cada una) y menos frecuente la formula digital AL (10\%). Con estos resultados se concluye que el IMC y la GC se encontró en los rangos aceptados para la edad y sexo; el somatotipo predominante en el grupo fue el mesomorfo balanceado.
\end{abstract}

Palabras Claves: Aptitud física, deporte, dermatoglifía, ejercicio, rendimiento atlético, somatotipo.

Abstract. The purpose of the present study was to determine the dermatoglyphic profile and somatotyping of a group of adolescents (age $=15.2 \pm$ 0.3) from the Córdoba soccer team (Colombia). Standardized anthropometric measurements were made (height, body weight, skinfolds, girths and breadths); indicators, such as body mass index (BMI), fat mass (FM), and somatotype were calculated from those measurements. At the same time, fingerprints were taken and digital designs were determined following the Cummins y Midlo (1942) protocol: Arches (A), Loops (L) and Whorls (W), Delta Index (D10), total number of lines on the right hand (SQTL), and additionally the types of digital formulas (AL, ALW, 10L, 10W, LW and WL). The results of the anthropometric indicators were: $\mathrm{BMI}=20.5 \pm 2.1 \mathrm{~kg}^{\prime}{ }^{\prime} \mathrm{m}^{2}, \mathrm{FM}=10.2 \pm 2.7 \%$; somatotype $=3.4 \pm 0.5-4.6 \pm 0.9-3.4 \pm 1$ (mesomorph - endomorph - ectomorph, respectively). Regarding the dermatoglyphic indicators, a frequency of: $\mathrm{A}=5 \%, \mathrm{~L}=65 \%$ and $\mathrm{W}=31 \% \mathrm{was}$ found; $\mathrm{D} 10=12.6 \pm 3.5$ and SQTL $=131.7 \pm 39.1$. On the other hand, the predominance of digital formulas were LW (40\%), followed by WL ( $20 \%$ ), ALW and 10L (with 15\% each) and less frequently the digital formula AL (10\%). Our results suggest that BMI and FM were found in the accepted ranges for age and sex; the predominant somatotype in the group was the balanced mesomorph.

Key Words: Physical fitness, sport, dermatoglyphic, exercise, athletic performance, somatotype.

\section{Introducción}

El Fútbol requiere de un análisis estructural que diferencie las capacidades y habilidades más fundamentales para un mayor rendimiento; para ello, se debe aplicar un conjunto de procedimientos de orden metodológico que identifiquen las regulaciones externas y destrezas que caractericen las posibilidades de éxito de cada jugador en el terreno de juego. De esta manera, el estudio del rendimiento deportivo, en deportes complejos como el futbol, demanda la identificación de variables con un impacto real en el resultado (Aguilar, 2018).

Es así como han surgido estudios de tipo antropométricos accesibles y con alto grado de validez, tendientes a tipificar la composición y la forma corporal de los deportistas (Jorquera Aguilera et al., 2013; Galaviz, Gutiérrez, Domínguez, \& Perea, 2018); Torreblanca-Martínez, Arráez, Otero-Saborido, \& González-Jurado, 2018), siendo el más claro ejemplo el estudio del somatotipo, definido como la cuantificación de la forma y composición del cuerpo humano. El somatotipo contempla en su estudio tres componentes: endomorfía, mesomorfía y la ectomorfía. La endomorfía, el primer componente, está relacionado con el nivel de adiposidad corporal y da a conocer atributos del aspecto físico tal como la redondez corporal; la mesomorfía, el segundo componente, describe el desarrollo musculoesquelético del cuerpo y además describe el aspecto físico tal como la robustez corporal, expresada en términos del desarrollo muscular y óseo; la ectomorfía, el tercer componente, expresa la delgadez relativa del cuerpo, describiendo en cuanto al aspecto físico la linealidad relativa del cuerpo, el poco desarrollo muscular o adiposo (Duquet \& Carter, 2009).

El método antropométrico propuesto por Heath \& Carter, para determinar el somatotipo, es el más comúnmente utilizado en la actualidad y se obtiene aplicando mediciones corporales para calcular los componentes del somatotipo (Carter, 2002; Rivera Sosa, 2005). En

Fecha recepción: 05-08-18. Fecha de aceptación: 13-12-18 Jorge Luis Petro

jlpetros@hotmail.com este sentido, se necesitan diez dimensiones antropométricas para calcular el somatotipo por el método antropométrico, que son: talla de pie, masa corporal, cuatro pliegues cutáneos (tríceps, subescapular, supraespinal, pierna medial), dos diámetros (húmero y fémur) y dos perímetros musculares de las extremidades del brazo (flexionado) y pantorrilla (Carter \& Heath, 1990; Carter, 2002)

Por otra parte, en la exploración de variables adicionales para estudiar el potencial de rendimiento atlético, complementario al estudio antropométrico o de la condición física, se han planteados métodos que intentan predecir el potencial deportivo desde temprana edad, tal como es el caso de la dermatoglifía, que es el estudio de patrones o indicadores dactilares obtenidos de las impresiones digitales de las manos y que han sido correlacionados con las capacidades físicas de los deportistas. En este sentido, se ha descrito que en la dermatoglifía aplicada al deporte, existen diseños de reconocimiento en la huella(Figura 1), como es el arco (A), presilla (L) y verticilo (W), que constituyen las características cualitativas (categóricas); asimismo, indicadores como la sumatoria total de líneas de los 10 dedos (SQTL) y la intensidad de los diseños (presencia de deltas en las huellas digitales) o el índice delta(D10) que constituyen las características cuantitativas (Fernandes Filho, 2010; Morales, 2014).0

De forma particular, se ha planteado que los diseños dactilares están relacionados con el potencial de desarrollo de las capacidades físicas (Juárez-Toledo, Domínguez-García, Laguna-Camacho, Sotomayor-Serrano, \& Balbás-Lara, 2018). De esta manera, se ha esti-

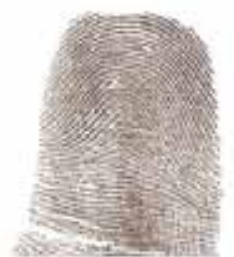

Arco (A)

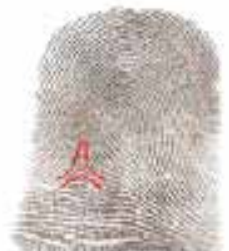

Presilla (L)

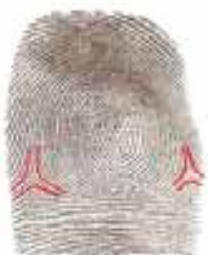

Verticilo (W)

Figura 1. Diseños o dibujos más comunes en la huella digital 
pulado que la presencia de A está relacionada con la capacidad de fuerza, la L con la capacidad de velocidad y el W con la coordinación motora (Fernandes Filho, 2010). Del mismo modo, elevados valores de SQTL y D10 han sido asociado con nivel de resistencia y coordinación motora (Fernandes Filho, 2010;Abramova, Nikitina, Shafranova, Kotchetkova, \& Secamova, 1996). Es así como en el análisis de estas características dermatoglíficas, autores como Abramova et al. (1996) han planteado clasificaciones de los índices somático-funcionales de atletas de alto rendimiento, llegando a configurarse cinco principales grupos (Tabla 1).

Bajo el marco de lo expuesto anteriormente, el objetivo del presente estudio fue establecer el perfil dermatoglífico y la somatotipificación de un grupo de jugadores adolescentes de fútbol, pertenecientes a la Selección Córdoba (Colombia), para lo cual se contempló la inclusión de una serie de procedimientos de orden antropométrico y estudio de parámetros de la huella dactilar que han sido relacionados con el potencial del rendimiento físico.

\section{Métodos}

\section{Sujetos}

Se evaluaron a 20 jugadores de futbol (edad, $15.2 \pm 0.3$ años; talla, $169.3 \pm 4.3 \mathrm{~cm}$; masa corporal, $58.7 \pm 5.0 \mathrm{~kg}$ ) que conformaban la selección masculina de fútbol del departamento Córdoba (Colombia). Estos jugadores participaron de un torneo preselectivo, como requisito previo, para conformar la selección mencionada y que es organizado por la Liga de Fútbol de Córdoba (Colombia). Los sujetos participaron de forma libre y voluntaria en este estudio (a través de un consentimiento informado firmado por los padres de familia) de conformidad con las normas bioéticas para la investigación, contemplada en la resolución No. 008430 de 1993 del Ministerio de Salud.

\section{Procedimientos}

Antropométrica y Somatotipificación. Se realizaron mediciones antropométricas de talla, masa corporal, pliegues cutáneos (tríceps, subescapular, supraespinal, abdominal, muslo y pierna), perímetros (brazo y pantorrilla) y diámetros (humero y fémur). Estas mediciones se realizaron siguiendo los protocolos establecidos por la Sociedad Internacional para el Avance de la Cineantropometría «ISAK (Stewart, Marfell-Jones, Olds, \& De Ridder, 2011). Con estas medidas se derivaron los indicadores antropométricos de estudio, tal como se describe a continuación.

$$
\text { Índice de Masa Corporal (IMC): } \frac{\text { Masa Corporal (kg) }}{\text { Talla (m) }}
$$

Porcentaje de Grasa Corporal (Slaughter et al., 1988) = $0.735 \mathrm{x}$ (Pliegue Tríceps + Pliegue Pierna Medial) + 1.0

Componentes del Somatotipo (método de Carter \& Heath, 1990):

Endomorfismo $=-0.7182+0.1451 \times$ ÓPC $-0.00068 \times$ ÓPC ${ }^{2}+$ $0.0000014 \times$ ÓPC $^{3}$

Donde, ÓPC = (suma de pliegues tricipital, subescapular y supraespinal) multiplicada por 170.18/talla (en cm). Esto representa el endomorfismo corregido por la talla.

Mesomorfismo $=(0.858 \times$ diámetro húmero $+0.601 \times$ diámetro fémur +0.188 x perímetro de brazo corregido +0.161 x perímetro de pantorrilla corregido) - (altura x 0.131$)+4.5$

Para calcular el ectomorfismo, de acuerdo con el Índice Ponderal (IP), se utilizan tres ecuaciones diferentes: si el IP es mayor o igual a 40.75, entonces: ectomorfismo $=0.732 \times$ IP -28.58 ; si el IP es menor que 40.75 y mayor a 38.25 , entonces: ectomorfismo $=0.463 \mathrm{x}$ IP 17.63; si el IP es igual o menor que 38.25, entonces: ectomorfismo = 0.1 (Carter, 2002).

Perfil Dermatoglífico. Se estableció mediante el protocolo de dermatoglifía propuesto por Cummins \& Midlo, (1942), donde se tomaron las huellas de los diez dedos de las manos de cada jugador, obteniéndose el dactilograma o dibujo dactilar. El registro de la huella
Tabla 1.

Clasificación del conjunto de los índices dermatoglíficos e índices somático-funcionales en atletas rusos de alto rendimiento. Clase D10 SQTL Características Funcionales

\begin{tabular}{ccccc}
\hline \multirow{2}{*}{ Clase } & \multirow{2}{*}{ D10 } & \multirow{2}{*}{ SQTL } & \multicolumn{2}{c}{ Características Funcionales } \\
\cline { 4 - 6 } I & 5.5 & 28 & $\begin{array}{c}\text { Estatura, Fuerza Absolumo } \\
\text { Coordinación Resistencia, }\end{array}$ & Fivel máximo \\
\hline II & 6.0 & 48 & Coordinación & Fuerza relativa \\
\hline III & 11.6 & 126 & Fuerza absoluta y fuerza relativa & Tamaño corporal \\
\hline IV & 13.1 & 134 & Tamaño corporal, Fuerza absoluta & Coordinación y Resistencia \\
\hline V & 17.5 & 163 & Fuerza relativa & Coordinación \\
\hline \multicolumn{2}{l}{ Fuente: Abramova et al. 1996} &
\end{tabular}

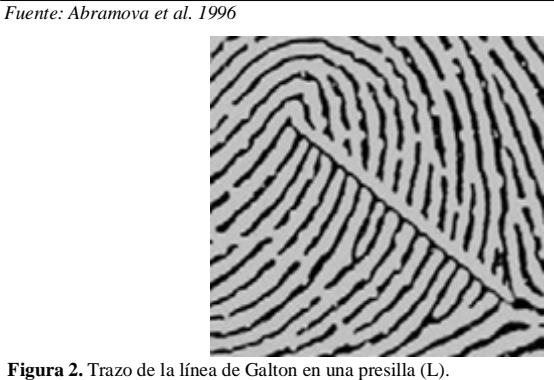

dactilar de los 10 dedos y las características dermatoglíficos fue realizado por un perito lofoscopita. De esta forma, se reconocieron los 3 principales diseños arco (A), presilla (L) y verticilo (W); posteriormente, se procedió a calcular el índice delta (D10) o intensidad de los deltas de los 10 dedos de las manos, donde por cada L representa un valor de 1 y por cada W serían 2 deltas (De Carvalho, Da Silva, \& Fernandes, 2005). En el D10 el valor mínimo es cero 0 y el valor máximo es 20, donde el valor 0 es justificado solo con la presencia de A y, por tanto, la ausencia de deltas. La fórmula para hallar el D10 es la siguiente:

D10 = ÓL + 2ÓW

Asimismo, se halló la sumatoria de la cantidad total de línea(SQTL) de todos los dedos en las dos manos. Para esto, se determinó el número de líneas existentes únicamente en el núcleo de las L y en el núcleo de los W por la línea de Galton(Figura 2), que es una línea imaginaria que tiene como punto de partida el delta y el dellegada el centro del núcleo, que se sitúa en el ápice (extremo superior o punta) de una L. De esta forma, se estableció la cantidad de líneas existentes en el dactilograma que están atravesadas por la línea de Galton, sin tener en cuenta el punto de partida (punto déltico) y el punto de llegada o punto nuclear (Zary, Reis, Rouboa, Silva, \& Fernandes, 2010).

Tal como se ha indicado, SQTL constituye una característica cuantitativa en el estudio de la Dermatoglifía(Da Cunha \& Fernandes Filho, 2004; Del Veccino \& Goncalves, 2011; Diaz \& Espinosa, 2008), siendo el indicador que tiene mayor posibilidad de comparaciones, en vista que varios estudios en el ámbito deportivo (e.g. en Rusia y Brasil) utiliza este indicador dermatoglífico (Veccino \& Goncalves, 2011).

Finalmente, se procede a establecer la frecuencia del tipo de fórmulas digitales, que indican la representación de los diferentes tipos de diseños: AL = Indica presencia de A y L (en cualquier combinación); ALW = La presencia de A, L y W (en cualquier combinación); 10L= Indica presencia de diez L; 10W = Indica presencia de diez W; LW = Presencia de Ly W, con la condición de que la frecuencia de L sea mayor o igual a 5; WL= Presencia de Wy W, pero con la condición de que la frecuencia de W sea mayor que cinco (Souza Menezes \& Fernandes Filho, 2006; Santos \& Fernandes Filho, 2003; Da Cunha \& Fernandes Filho, 2004)

\section{Análisis Estadístico}

El análisis estadístico se realizó con el software SPSS versión 20 (Chicago, IL, USA). El procedimiento estadístico fue conducido principalmente a la caracterización descriptiva de las variables de estudio (somatotipo y dermatoglifía), expresando los resultados como frecuencias (recuento y porcentajes) o como media y desviación estándar. No obstante, se realizó procedimientos estadísticos de rigor como el contraste de normalidad de los datos con el Test de Shapiro-Wilk. Para presentar un perfil de las principales variables de interés de este estudio, el somatotipo (componentes de la endomorfía mesomorfía y ectomorfía), los diseños dactilares y la SQTL y el D10, los valores 
fueron ajustados (normalizados) en una escala de 0 - 1 con la siguiente formula (Nogueira, da Cunha Junior, Dantas, \& Fernandes Filho, 2005; Souza Menezes, 2006; Medellin, 2014):

\section{valor observado - valor mínimo}

\section{valor máximo -val or mínimo}

Posteriormente, se establecen los intervalos de confianza de 95\% para la media. Estos tres valores (límite inferior, media y límite superior) son representado en un gráfico de radar para la caracterización, de forma visual, de las variables dermatoglíficas y del somatotipo (Medina \& Fernandes Filho, 2002; Zary et al., 2010).

\section{Resultados}

Los resultados derivados del análisis estadístico de los datos antropométricos, incluyendo los componentes del somatotipo (endomorfía, mesomorfía y ectomorfía), son presentados en la Tabla 2 según la posición de juego de los sujetos de estudio y el total (promedio del equipo).

Tabla 2.

Resultados de los indicadores antropométricos básicos y componentes del somatotipo. Talla (kg) MCT (kg) $\quad \begin{gathered}\text { IMC } \\ \left(\mathrm{kg} \cdot \mathrm{m}^{2}\right)\end{gathered}$ GC (\%) Endomorfía MesomorfíaEctomorfía

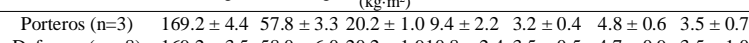

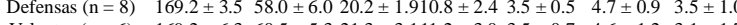

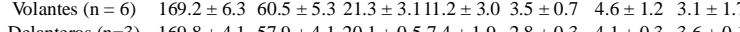

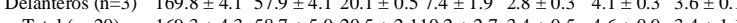

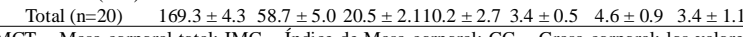
MCT = Masa corporal total; IMC = Índice de Masa corporal; GC = Grasa corporal; los valore son expresados en media \pm desviación estándar

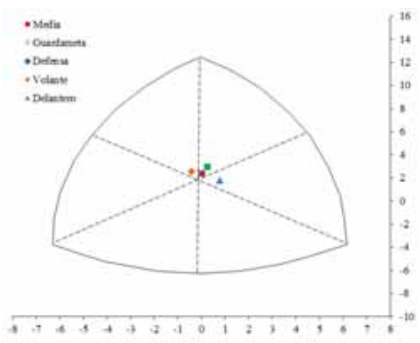

Figura 2. Ubicación del somatotipo en la somatocarta

Tabla 3.

\begin{tabular}{cccccc}
\multicolumn{7}{l}{ Resultados característicos dermatoglíficas. } \\
\hline & $\mathrm{A}(\%)$ & $\mathrm{L}(\%)$ & $\mathrm{W}(\%)$ & $\mathrm{D} 10(\mathrm{X} \pm \mathrm{DE})$ & $\mathrm{SQTL}(\mathrm{X} \pm \mathrm{DE})$ \\
\hline Porteros $(\mathrm{n}=3)$ & 3 & 57 & 40 & $13.7 \pm 4.0$ & $132.7 \pm 28.2$ \\
Defensas $(\mathrm{n}=8)$ & 8 & 61 & 31 & $12.4 \pm 4.1$ & $132.4 \pm 53.6$ \\
Volantes ( $\mathrm{n}=6)$ & 0 & 70 & 30 & $13.0 \pm 3.3$ & $131.8 \pm 33.6$ \\
Delanteros (n=3) & 7 & 73 & 20 & $11.3 \pm 3.5$ & $128.3 \pm 29.3$ \\
Total (n=20) & 5 & 65 & 31 & $12.6 \pm 3.5$ & $131.7 \pm 39.1$ \\
\hline $\mathrm{X}=$ Media; DE = Desviación estándar & & &
\end{tabular}

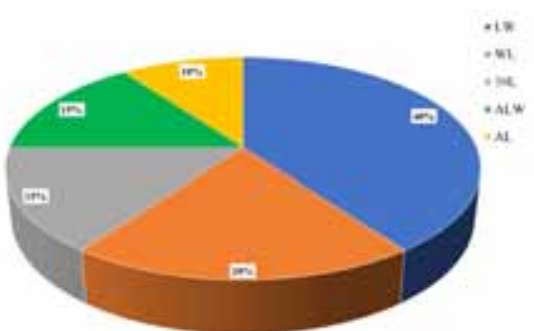

Figura 3. Distribución de las fórmulas digitales

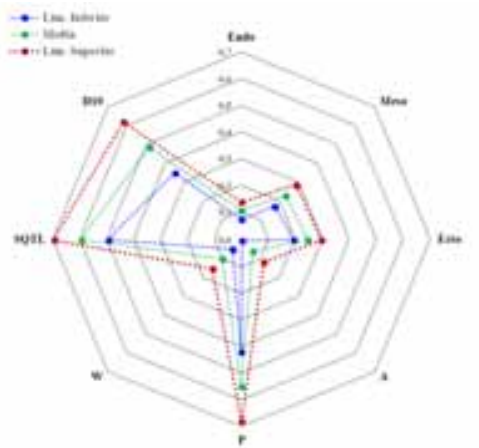

Figura 4. Valores normalizados de los componentes del somatotipo y de las características dermatológicas.
Para complementar el estudio de la caracterización del somatotipo, se muestra la representación del somatotipo en la somatocarta (Figura 2).

En lo que corresponde a los resultados de las características dermatoglíficas (Tabla 3), el equipo de adolescentes muestra una distribución de $\mathrm{L}$ de una media de $6.5 \pm 2.8$ (65\%), de W $3.1 \pm 3.1$ (31\%) y deA $0.5 \pm 0.9$ (5\%). Es de resaltar, como punto de interés, que la mayor presencia de Ly A fue en los delanteros (73\% y 7\%, respectivamente), mientras que los porteros registraron mayor W (40\%) y en los volantes no se registró diseños A. Por otra parte, en cuanto a la característica cuantitativa de las dermatoglifía, la SQTL fue $131.7 \pm 39.1$ y el D10 fue de $12.6 \pm 3.5$.

En cuanto al tipo de fórmulas digitales, se encontró que la mayor distribución fue la de LW (40\%), seguido de WL (20\%), ALW (15\%), 10L (15\%)y, finalmente, AL(10\%), tal como se muestra en la Figura 3.

Los indicadores de los componentes del somatotipo y de la dermatoglifía, son caracterizados en el gráfico de radar (Figura 2).

\section{Discusión}

El análisis de indicadores antropométricos como la masa corporal, la talla y el IMC, permiten conocer aspectos relacionados con el estado nutricional, estatus de crecimiento o el desarrollo físico en niños y adolescentes. En tal sentido, de acuerdo con De Onís et al. (2007), el IMC de estos adolescentes se hallan dentro de los valores normales para la edad y sexo, en vista que estos se encontraron dentro del punto de corte de normalidad (e» -1 a d»1 desviación estándar) establecido por la OMS y que son adoptados en Colombia, como patrones de referencia y puntos de corte para la clasificación antropométrica del estado nutricional en menores de 18 años (Ministerio de Protección Social, 2016). El porcentaje de grasa corporal, de forma general, se puede decir que estuvo dentro de los rangos aceptados, según valores de referencia para la edad y sexo (Moreno et al., 2005; Widiyani et al., 2011; Laurson, Eisenmann, \& Welk, 2011).

Con respecto al estudio del somatotipo, el análisis estadístico mostró que el componente que predominó fue el de mesomorfía, seguido por la ectomorfía y la endomorfía, clasificándose el grupo evaluado como mesomorfo balanceado; es decir, el componente mesomórfico (relacionado con el desarrollo musculoesquelético) es dominante, mientras que el endomórfico y el ectomórfico no tienen una diferencia mayor (Carter \& Heath, 1990). De acuerdo con estos resultados, el grupo estudiado presenta un desarrollo musculoesquelético relativo (mesomorfía de 4.6 \pm 0.9 ), una adiposidad relativa moderada (endomorfía de $3.4 \pm 0.5$ ) y una linealidad relativa moderada (ectomorfía de $3.4 \pm$ 1.1).

Comparados estos resultados con otros estudios realizados en adolescentes de este deporte, como el realizado por Godoy-Cumillaf et al. (2015) fueron similares: predominio del componente mesomórfico; sin embargo, se recalca que el componente de ectomorfía fue más bajo en el estudio citado (2.3 \pm 1.8 ) en comparación con el nuestro (3.4 \pm 1.1$)$. De la misma manera, el estudio realizado por Albornoz (2014), en futbolistas adolescentes, también encontró que el somatotipo predominante fue el mesomorfo balanceado (2.5 - 3.9 - 2.9). Por otra parte, considerando la investigación de Castanhede, Fernandes, \& Dantas (2003), donde evaluaron a 48 jugadores de la liga de fútbol profesional de Brasil, que compitieron campeonatos en ese país durante el 20012002, hallaron que el somatotipo predominante fue el mesomorfo balanceado (2.7-4.8-2.3), siendo esto el mismo concepto valorativo que se encontró en el presente estudio.

Por otro lado, un aspecto en el cual se enfocó este estudio es la utilización de la dermatoglifía como medio para identificar el potencial de rendimiento deportivo. En cuanto a esto, se pudo establecer que el grupo de adolescentes futbolistas presentan una distribución importantede $\mathrm{L}$, configurándose un patrón de los diseños dermatoglíficos $\mathrm{L}>\mathrm{W}>\mathrm{A}$. Sin embargo, esto es un planteamiento que, expresado así, da poca información sobre las particularidades de poblaciones específicas, como grupos de disciplinas deportivas (comparadas con grupos de referencia 
o de control).

Comparado con estudios de poblaciones de referencia (futbolistas profesionales), como los realizados por Castanhede et al. (2003), la frecuencia de A(vinculada a la fuerza), L (asociada a la velocidad) y D10 (vinculada a la coordinación motora) es casi similar; no obstante, en el presente estudio se halló un mayor recuento de W (ligada a la coordinación motora) y de la SQTL (asociada con la resistencia y coordinación). Igualmente, comparados con los resultados reportados por Abramova et al. (1996), el equipo evaluado en este estudio tuvo un mayor recuento de W, D10 y de SQTL, pero una menor distribución de los diseños A y L. Siguiendo en esta línea, según el estudio realizado por HernándezMosqueira et al. (2013) en jugadores de futbol adolescentes de futbol de la ciudad Chillan (Chile), documentaron una mayor frecuencia de A, pero una menor prevalencia de L, W, D10 y SQTL en comparación con nuestros resultados. Es notable que el estudio de Silva et al. (2008), fue el que reportó la mayor SQTL de las investigaciones aquí contempladas para la comparación de características dermatoglíficas en el futbol, mientras que el Abramova et al. (1996) reporto la mayor distribución de W (Tabla 4).

\begin{tabular}{|c|c|c|c|c|c|}
\hline Estudio & A & $\mathrm{L}$ & $\mathrm{W}$ & D10 & SQTL \\
\hline Abramova et al. (1996) & 3.2 & 62.7 & 34.1 & 13.4 & 142.6 \\
\hline Castanhede et al. (2003) & 5.8 & 69.0 & 25.6 & 12.0 & 99.2 \\
\hline Silva et al. (2008) & 8.0 & 60.0 & 31.3 & 12.3 & 181.2 \\
\hline Hernández-Mosqueira (2013) & 19.0 & 57.0 & 24.0 & 9.6 & 117.0 \\
\hline Presente estudio & 5.0 & 65.0 & 30.0 & 12.6 & 131.7 \\
\hline Media & 8.2 & 62.74 & 29.0 & 12.0 & 134.3 \\
\hline
\end{tabular}

Según los resultados obtenidos y lo documentado en la dermatoglifía, el perfil dermatoglífico del equipo evaluado en el presente estudio, esta direccionado hacia el desarrollo de la velocidad, la resistencia y la coordinación, sustentado ello en la frecuencia de L, W y a los valores de SQTL y D10; además de la distribución de los tipos de fórmulas digitales encontradas $\mathrm{LW}>\mathrm{WL}>\mathrm{ALW}>10 \mathrm{~L}>\mathrm{AL}$. Con base en estos resultados, este perfil somatotípico y dermatoglífico está relacionado con los requerimientos fisiológicos del fútbol, caracterizado por las deacciones acíclicas e intermitente (González, Suárez-Arrones, Bretones, \& de Villarreal, 2018); Rojas-Inda, 2018), donde se ven involucradas diversas capacidades físicas como la velocidad, la resistencia y la coordinación motriz. Esto representa un potencial que tiene el grupo evaluado (hacia el futbol profesional).

Finalmente, proponemos que la dermatoglifía es un método que puede sumarse a los demás procedimientos convencionales (como la antropometría/cineantropometría los test de aptitud física) para la evaluación de la detección y selección del potencial del rendimiento deportivo, teniendo los alcances y limitaciones de este método a la luz de la evidencia actual.

\section{Conclusiones}

Los indicadores antropométricos de masa corporal, talla y el índice de masa corporal y grasa corporal se encuentran dentro de los valores de normalidad para la edad y sexo. El estudio del somatotipo estableció que el equipo de estudio se clasifica como mesomorfo balanceado (3.4 \pm $0.5-4.6 \pm 0.9-3.4 \pm 1.1$ ); asimismo, los tres componentes del somatotipo se clasifican, de acuerdo con el valor encontrado, como moderado.

La caracterización de la dermatoglifía estableció que la frecuencia de los diseños de las huellas dactilares fue L (65\%), W (31\%), A(5\%) y un moderado recuento del D10 (12.6 \pm 3.5$)$ y SQTL (131.7 \pm 39.1$)$ comparados con estudios realizados en futbolistas. El tipo de fórmula digital que predominó fue la LW (40\%), seguido de WL (20\%) y ALW y 10L (15\% para cada una), siendo menos frecuente la formula digital AL (10\%). De acuerdo con el perfil dermatoglífico encontrado, y con base en los valores de referencias disponible en la literatura actual, el equipo evaluado tiene potencial hacia el desarrollo de la resistencia, la velocidad y la coordinación motriz y, paralelamente, con menos predisposición a la fuerza máxima.
Los autores del presente estudio plantean que la dermatoglifía puede ser tenida en cuenta como un medio más para evaluar el potencial deportivo, que podría ser considerada dentro de las baterías de test existentes para la selección y detección del talento deportivo; sin embargo, hay la necesidad de seguir explorando los alcances y las limitaciones de este método (contrastándola con otras técnicas de evaluación a nivel genético o genómico). Adicionalmente, se requieren establecer tablas de referencia más específicas o completas para valorar el perfil dermatoglífico asociado al rendimiento deportivo en deportes como el futbol.

\section{Referencias}

Abramova, T., Nikitina, T., Shafranova, E., Kotchetkova, N., \& Secamova, G. (1996). Finger Dermatoglyphs as Markers of the Funtional Features. (V. Rogozkin, y R. Maughan, Edits.) New York, United States: Plenum Press.

Aguilar, M.P. (2018). Temporal consistencies in two champion teams of European football? Retos: nuevas tendencias en educación física, deporte y recreación, (34), 94-99

Albornoz, V. (2014). Análisis de las características antropométricas del plantel de sub 15 del Club Atlético Juventud de Las Piedras [en línea]. Trabajo final de posgrado. Universidad Nacional de La Plata. Facultad de Humanidades y Ciencias de la Educación. En Memoria Académica. Recuperado de: http://www.memoria.fahce.unlp.edu.ar/ tesis/te.1003/te.1003.pdf.

Carter, J. (2002). The heath-carter anthropometric somatotype. Instructionmanual. Department of Exercise and Nutritional Sciences. San Diego, United States: San Diego State University Press., 1-26. Recuperado de: http://www.somatotype.org/HeathCarterManual.pdf

Carter, J., \& Heath, B. (1990). Somatotyping Development and Applications. New York, United States: Cambridge University Press.

Castanhede, A., Fernandes, J., \& Dantas, P. (2003). Dermatoglyphic and Somatotype profile of male soccer athletes of high performance in Rio de Janeiro-Brazil. Journal of Fitness and Performance, 2(4), 234-239.

Cummins, H., \& Midlo, CH. (1942). Palmar and plantar dermatoglyphics in prima-tes. Philadelphia.

Da Cunha, R., \& Filho, J. (2004). Identification of dermatoglyphic profile of high level foreign fencers of the three armed force, participants of fencing world championship-Havana-Cuba/ 2003. Fitness y Performance Journal, 3(5), 247-253.

De Carvalho, E., Da Silva, J., \& Fernandes, F. (2005). Dermatoglyphic, somatotypic and physiologic profiles of athletes of high performance, participants of resistance race, in Rio de Janeiro. Fitness y Performance Journal, 4(3), 168-174.

De Onís, M., Onyango, A., Borghi, E., Siyam, A., Nishida, C., \& Siekmann, J. (2007). Development of a WHO growth reference for school-aged children and adolescents. Bulletin of the World Health Organization,, 85(9), 660-667. doi:10.2471/BLT.07.043497.

De Souza Menezes, L., \& Fernandes Filho, J. (2006). Identificação e comparação das caracterísicas dermatoglíficas, somatotípicas e de qualidades físicas básicas de atletas de ginástica rítmica de diferentes níveis de qualificação esportiva. Fitness y performance journal, (6), 393-401.

Del Vecchio, F. B., \& Gonçalves, A. (2011). Dermatoglifos como indicadores biológicos del rendimiento deportivo. Revista andaluza de Medicina del Deporte, 4(1), 38-46.

Duquet, W., \& Carter, L. (2009). Somatotyping. En R. Eston, T. Reilly, R. Eston, y T. Reilly (Edits.), Kinanthropometry and exercise physiology laboratory manual: tests, procedures and data. New York, USA and Canada: Routledge.

Fernandes Filho, J. (2010). La impresión digital (dermatóglifos) y la detección de talentos deportivos. Curso de Dermatoglifia Deportiva. Memorias. Soacha, Colombia: Universidad de Cundinamarca. Galaviz, U. Z., Gutiérrez, A. O., Domínguez, I. D. J. T., \& Perea, R. H. 
(2018). Somatotipo en futbolistas mexicanos profesionales de diferente nivel competitivo. Retos: nuevas tendencias en educación física, deporte y recreación, (34), 100-102.

Godoy-Cumillaf, A., Valdés-Badilla, P., Salvador-Soler, N., CarmonaLópez, M., \& Fernández-Filho, J. (2015). Características Antropométricas de Adolescentes Pertenecientes a Distintas Escuelas Deportivas Formativas. International Journal of Morphology, 33(3), 1065-1070.

González, J. R., Suárez-Arrones, L., Bretones, A. R., \& de Villarreal, E. S. (2018). Efectos a corto plazo de un programa de entrenamiento de sobrecarga excéntrica sobre el rendimiento físico en jugadores de fútbol de élite U-16. Retos: nuevas tendencias en educación física, deporte y recreación, (33), 106-111.

Hernández, C., Hernández, D., \& Fernandes, J. (2013). Perfil dermatoglifico de jugadores profesionales de futbol del Club Deportivo Ñublense de la Ciudad de Chillan. Revista Motricidad Humana, 14(1), 9-15.

Hernández, C., Ibarra, J., Retamales MuñozF., Valenzuela R., Hernandez D., \& Fernandes Filho, J. (2013). Perfil dermatoglifico en futbolistas de proyección del club deportivo ñublense de chillan. Revista de las Ciencias de la Actividad Física del Instituto Nacional del Deporte. (8), 69-78.

Jorquera Aguilera, C., Rodríguez Rodríguez, F., Torrealba Vieira, M. I., Campos Serrano, J., Gracia Leiva, N., \& Holway, F. (2013). Características antropométricas de futbolistas profesionales chilenos. International Journal of Morphology, 31(2), 609-614.

Juárez-Toledo, L., Domínguez-García, M. V., Laguna-Camacho, A., Sotomayor-Serrano, N., \& Balbás-Lara, F. (2018). Somatotype and digital dermatoglyphic in mexican football players. Revista Internacional de Medicina y Ciencias de la Actividad Física y del Deporte, 18(70).

Laurson, K. R., Eisenmann, J. C., \& Welk, G. J. (2011). Body fat percentile curves for US children and adolescents. American journal of preventive medicine, 41(4), S87-S92.

Medellín, J. P. (2014). Caracterización dermatoglífica de las ciclistas colombianas de pista de altos logros en pruebas de semifondo. Revista UDCAActualidad y Divulgación Científica, 17(1).

Medina, M., \& Fernandes, J. (2002). Genetic and somatotype profiles identification of high performance male adult volleyball players in Brazil. Fitness y Performance Journal, 1(4), 12-19.

Ministerio de Protección Social (2016). Resolución Número de 2016 Recuperado de: https://www.icbf.gov.co/sites/default/files/ resolucion_no._2465_del_14_de_junio_de_2016.pdf.

Ministerio de Salud. Resolucion némero 8430 de 1993. Recuperado de: https://www.minsalud.gov.co/Normatividad_Nuevo/ RESOLUCION\%208430\%20DE\%201993.pdf

Morales, S. (2014). Genética deportiva. Atlantic International University. Recuperado de: https://www.aiu.edu/applications/ DocumentLibraryManager/upload/1-1282014-18272810487564.pdf
Moreno, L. A., Mesana, M. I., González-Gross, M., Gil, C. M., Fleta, J., Wärnberg, J., Ruiz, J., Sarría, A., Marcos, A., \& Bueno, M. (2006). Anthropometric body fat composition reference values in Spanish adolescents. The AVENA Study. European journal of clinical nutrition, 60(2), 191.

Nogueira, T. N., Da Cunha Junior, A. T., Dantas, P. M. S., \& Fernandes Filho, J.(2005). Perfil somatotípico, dermatoglífico e das qualidades físicas da seleção brasileira de handebol feminino adulto por posição de jogo. Fitness y performance journal, (4), 236-242.

Rivera Sosa, J. M. (2006). Valoración del somatotipo y proporcionalidad de futbolistas universitarios mexicanos respecto a futbolistas profesionales. Revista Internacional de Medicina y Ciencias de la Actividad Física y del Deporte, 6(21).

Rojas-Inda, S. (2018). analysis of internal and external load in small games in young football players. Revista Internacional de Medicina y Ciencias de la Actividad Física y del Deporte, 18(71).

Santos, M., \& Fernandes Filho, J. (2003). Perfis dermatoglífico, somatotípico e das qualidades físicas básicas dos pára-quedistas do exército brasileiro do ano de 2003. Fitness y Performance Journal, 3(2), 88-97.

Silva, I., Vianna, M., Gomes, A., \& Dantas, E. (2008). Diagnóstico do potencial genético físico e somatotipia de uma equipe de futebol profissional Fluminense. Revista Brasileira de Futebol, 1(1), 4958.

Slaughter, M., Lohman, T., Boileau, R., Horswill, C., Stillman, M., Van Loan, M., y Bemben, D. (1988). Skinfold equations for estimation of body fatness in children and youth. Human Biology, 60(5), 709723.

Souza Menezes, L. D. (2006). Identificação e comparação das características dermatoglíficas, somatotípicas e qualidades físicas básicas de atletas de GRD de diferentes níveis de qualificação esportiva. Fitness y Performance Journal, 5(6).

Stewart, A., Marfell-Jones, M., Olds, T., y De Ridder, H. (2011). International standards for anthropometric assessment. Lower Hutt, New Zeland: International Society for Advancement of Kinanthropometry.

Torreblanca-Martínez, V., Arráez, A., Otero-Saborido, F. M., \& González-Jurado, J. A. (2018). Características antropométricas y de rendimiento físico en futbolistas sub-11 según el nivel competitivo (Anthropometric and fitness performance characteristics in under-11 football players by competitive level). Retos: nuevas tendencias en educación física, deporte y recreación,(34), 146-149.

Widiyani, T., Suryobroto, B., Budiarti, S., y Hartana, A. (2011). The growth of body size and somatotype of Javanese children age 4 to 20 years. HAYATI Journal of Biosciences, 18(4), 182-192.

Zary, J. C., Reis, V. M., Rouboa, A., Silva, A. J., \& Fernandes Filho, J. (2010). The somatotype and dermatoglyphic profiles of adult, junior and juvenilemaleBrazilian top-level volleyball players. Science y Sports, 25(3), 146-152.
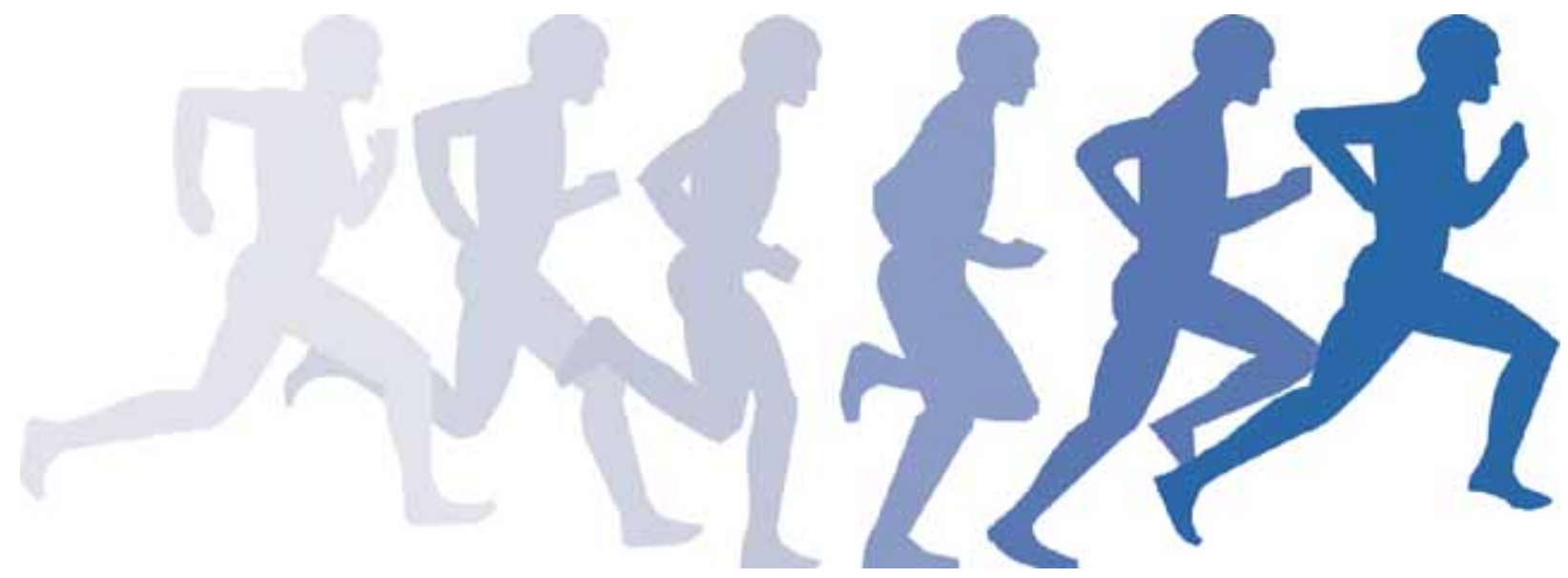